\title{
Enhanced $\mathrm{MgF}_{2}$ and LiF Over-coated Al Mirrors for FUV Space Astronomy
}

\author{
By
}

Manuel Quijada 


\section{Outlines}

$>$ Motivation: The need for better performing coatings in the Far-Ultraviolet (FUV)

D Enhanced UV coating development for the Thermospheric Temperature Imager (TTI)

$>$ More recent research efforts:

$>$ Preparation of 2-meter coating chambers

$>$ Study of other low-absorptions dielectric coatings for FUV applications

$>$ Conclusions

$>$ Acknowledgements 


\section{Enhanced FUV Coating Applications}

- Distant and faint objects are typically searched for in cosmic origin studies:

o Origin of large scale structure

- The formation, evolution, and age of galaxies

$\circ$ The origin of stellar and planetary systems

- Astronomical observations in the Far Ultraviolet (FUV) spectral region are some of the more challenging

- Very limited option of reflecting coatings to use at FUV wavelengths:

- Modest reflectivity offered by those coatings

○ $\mathrm{Al}+\mathrm{MgF}_{2}$ [typically $82 \%$ at Lyman-alpha, $1216 \AA$ ) that are used on reflecting surfaces of FUV instrumentation

- Improved reflective coatings for optics at FUV could yield dramatically more sensitive instruments .

- Permit more instrument design freedom 


\section{Optical Coating Deposition Processes}

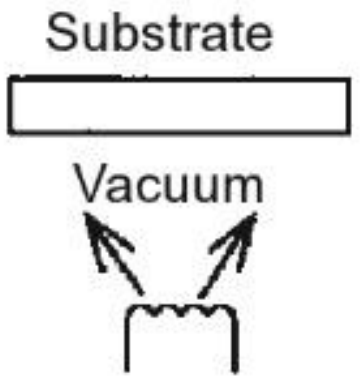

Filament

(a)

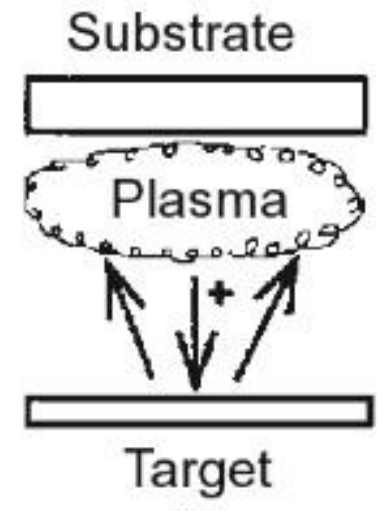

(b)

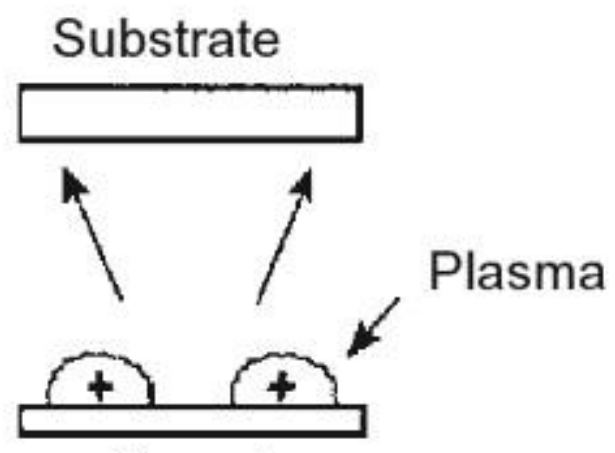

Target

(c)

\section{PVD}

- Material is heated until it reaches vapor form

- Material is deposited on the substrate where it condenses

- Typical deposition rates are 10-100 Å/Sec.

\section{Sputtering}

- Non-thermal evaporation process

- Atoms from a target are ejected by momentum transfer from energetic atom-size particles

- Particles are energized by an ion gun

- Deposition rate are much lower than PVD 1-5 Å/Sec. 


\section{Solidification vs. Crystallization}
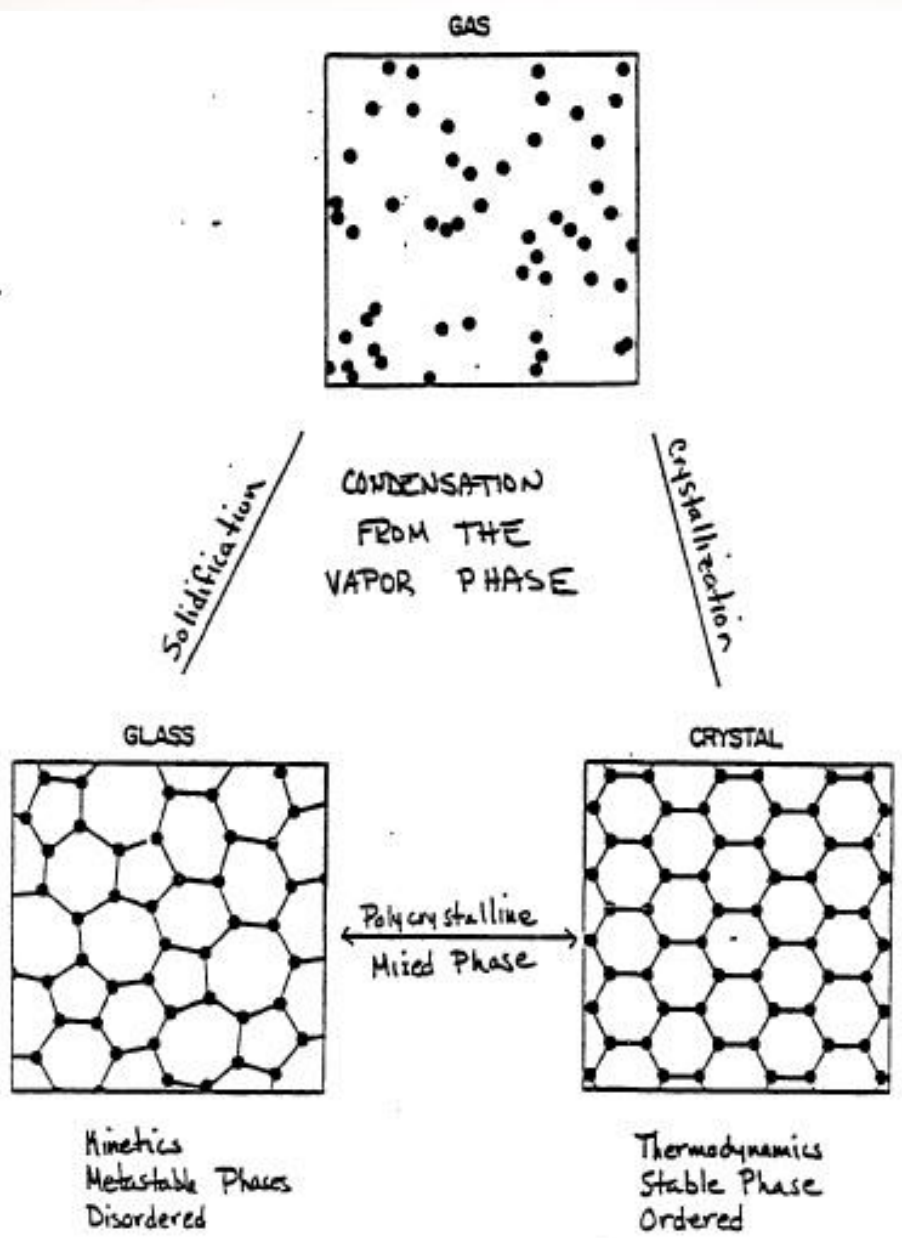


\section{TTI Mission Concept}

\section{TTI Optics}

- Imager to provide global-scale remote measurements of thermospheric temperature profile of atomic oxygen at an altitude of 200-400 KM

- Wavelength range: 135.4 to $135.6 \mathrm{~nm}(0.0004 \mathrm{~nm}$ resolution)

- Fabry-perot Etalon cavity:

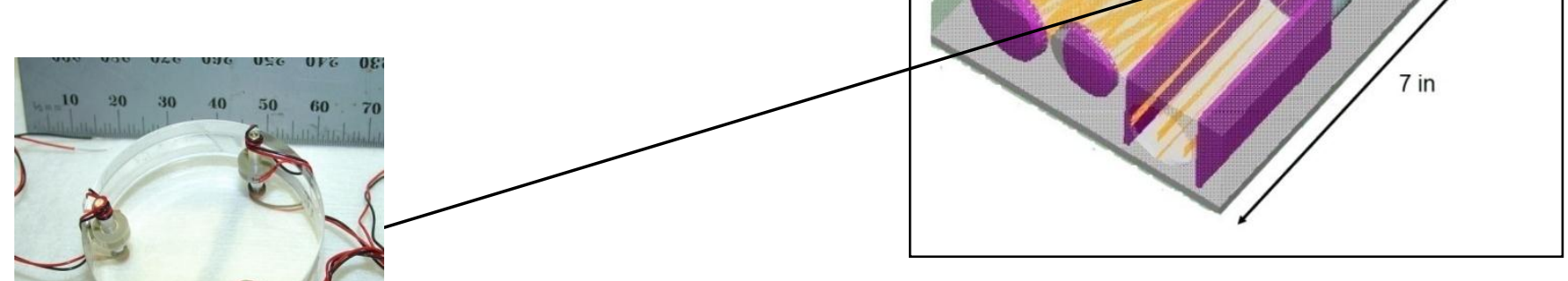

- GSFC effort to develop $\mathrm{Al}+\mathrm{MgF} 2$ coatings to meet requirements:

- High reflectance \& low absorption 


\section{High-Temperature Deposition Al+MgF2}

\section{3-step coating process:}

$\checkmark$ Al coat the substrate at room temperature to the planned layer thickness

$\checkmark$ As soon as possible after the Al deposition, overcoat the Al layer and substrate at room temperature with a thin 4$5 \mathrm{~nm}$ layer of $\mathrm{MgF}_{2}$ in order to protect the Al from oxidation and contamination.

$\checkmark$ Heat the substrate to maximum temperature and overcoat the thin MgF2, Al, and substrate with the planned thickness of MgF2.

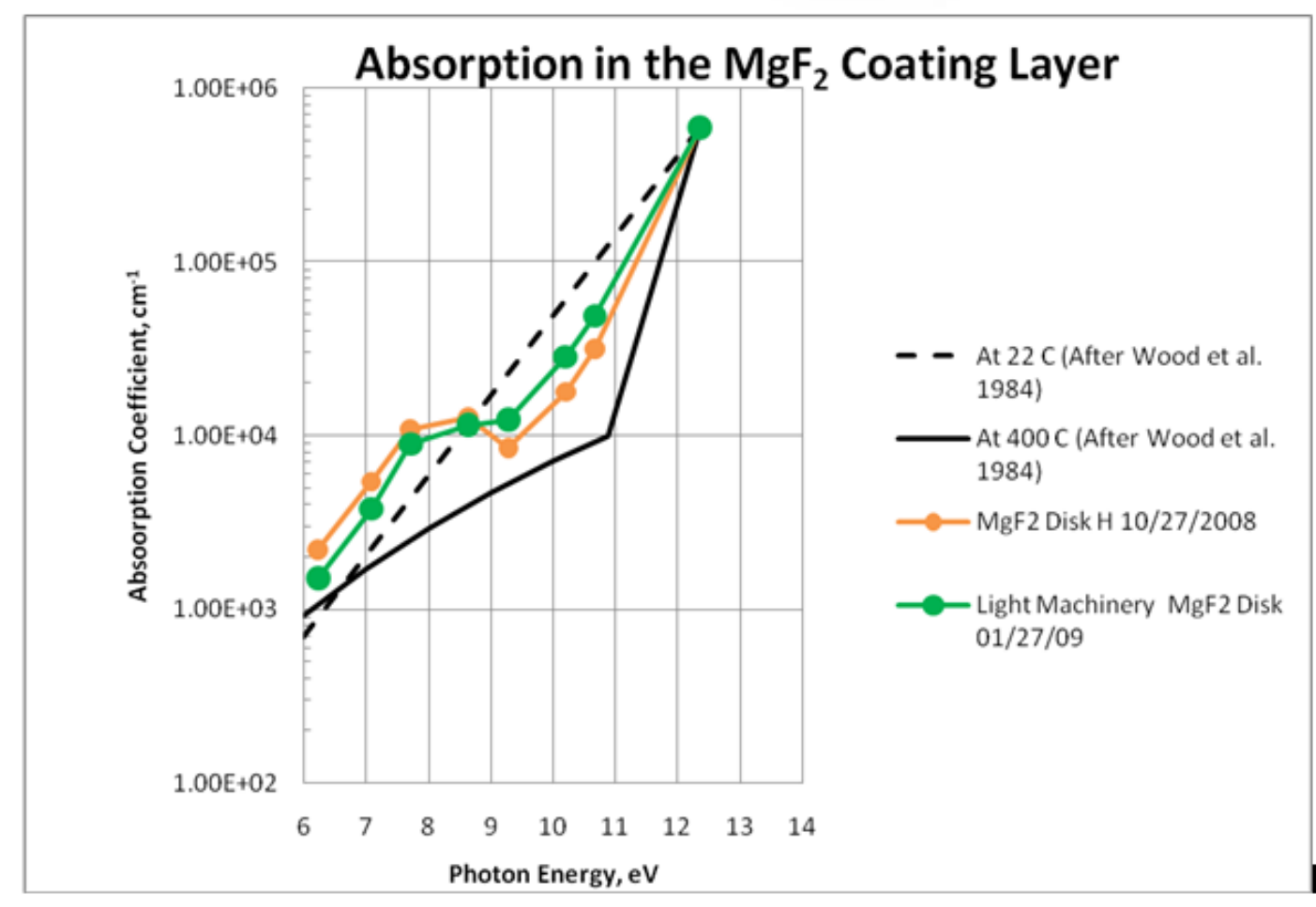




\section{TTI Etalon Plate Performance}

\section{Reflectance}

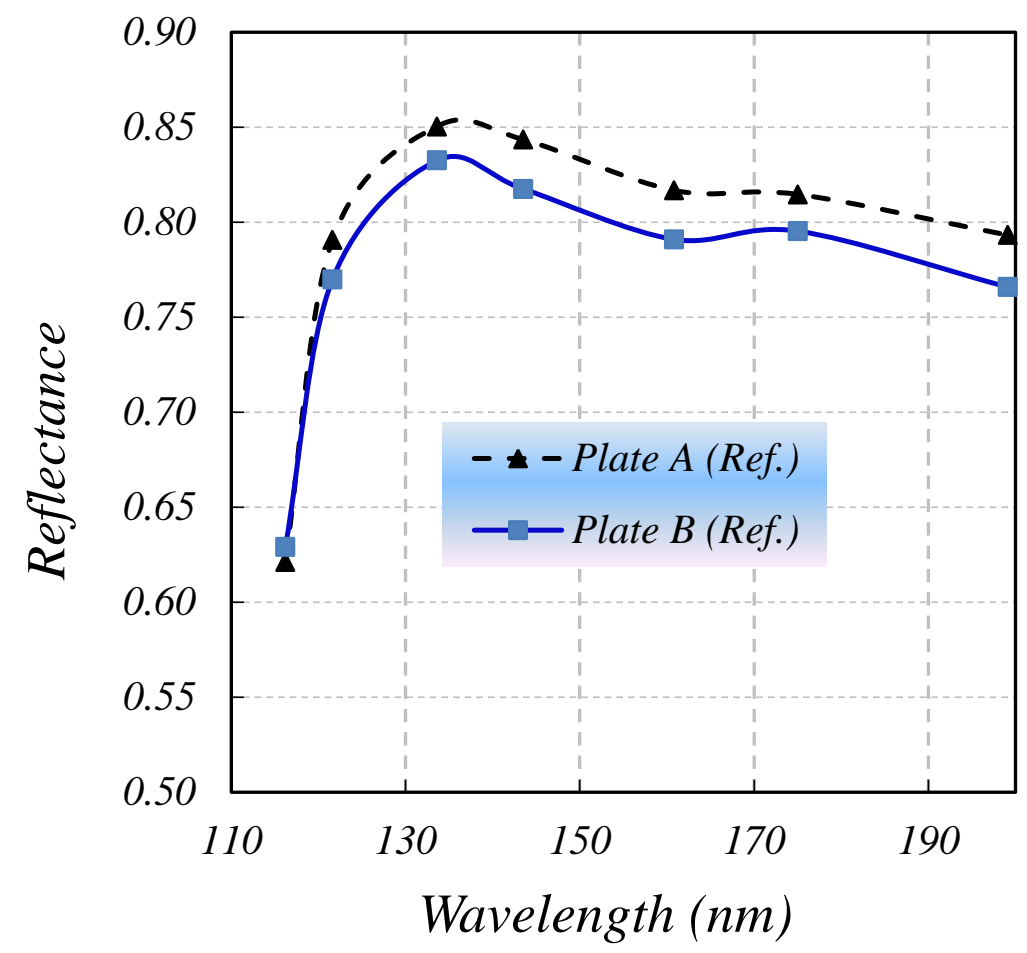

TTI Etalon plate 6a (Thicknesses: Al 277 A; MgF2 300A)
Transmittance

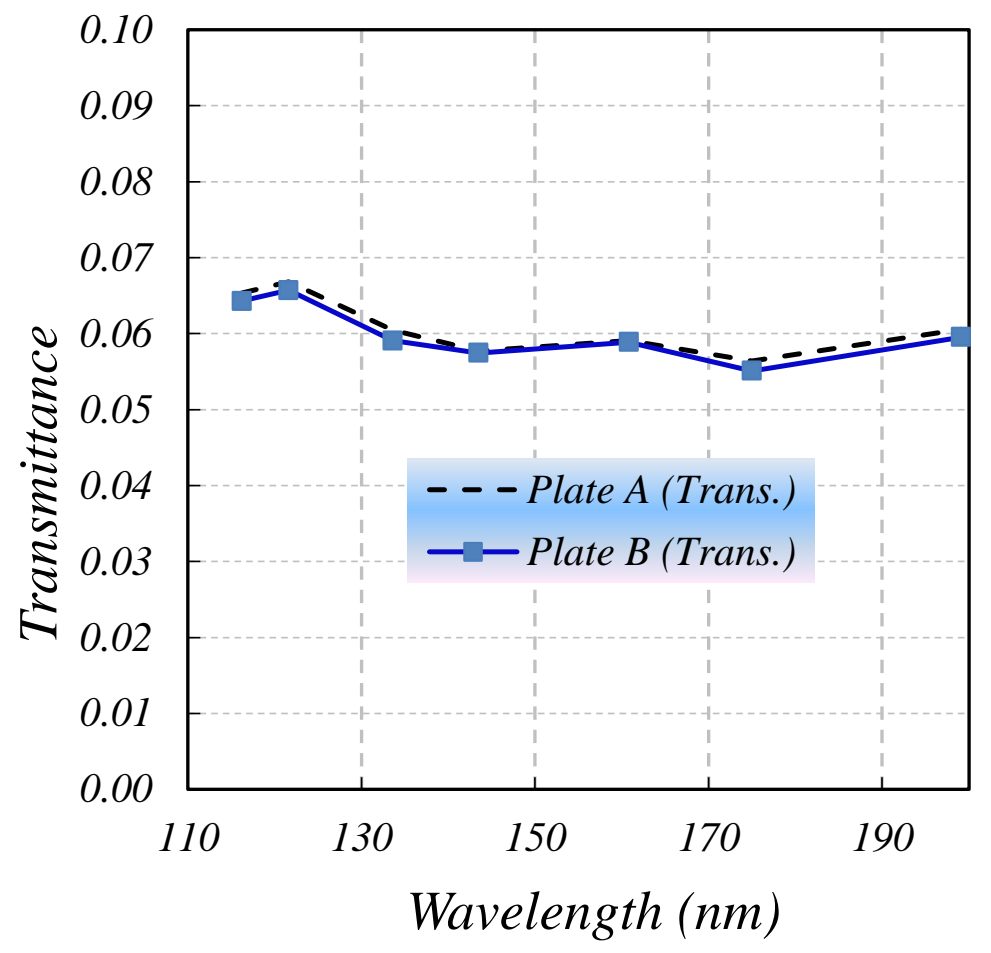

TTI Etalon plate 6b (Thicknesses: Al 278 A; MgF2 310A) 


\section{Evaporated Al+MgF2 Mirror Performance}

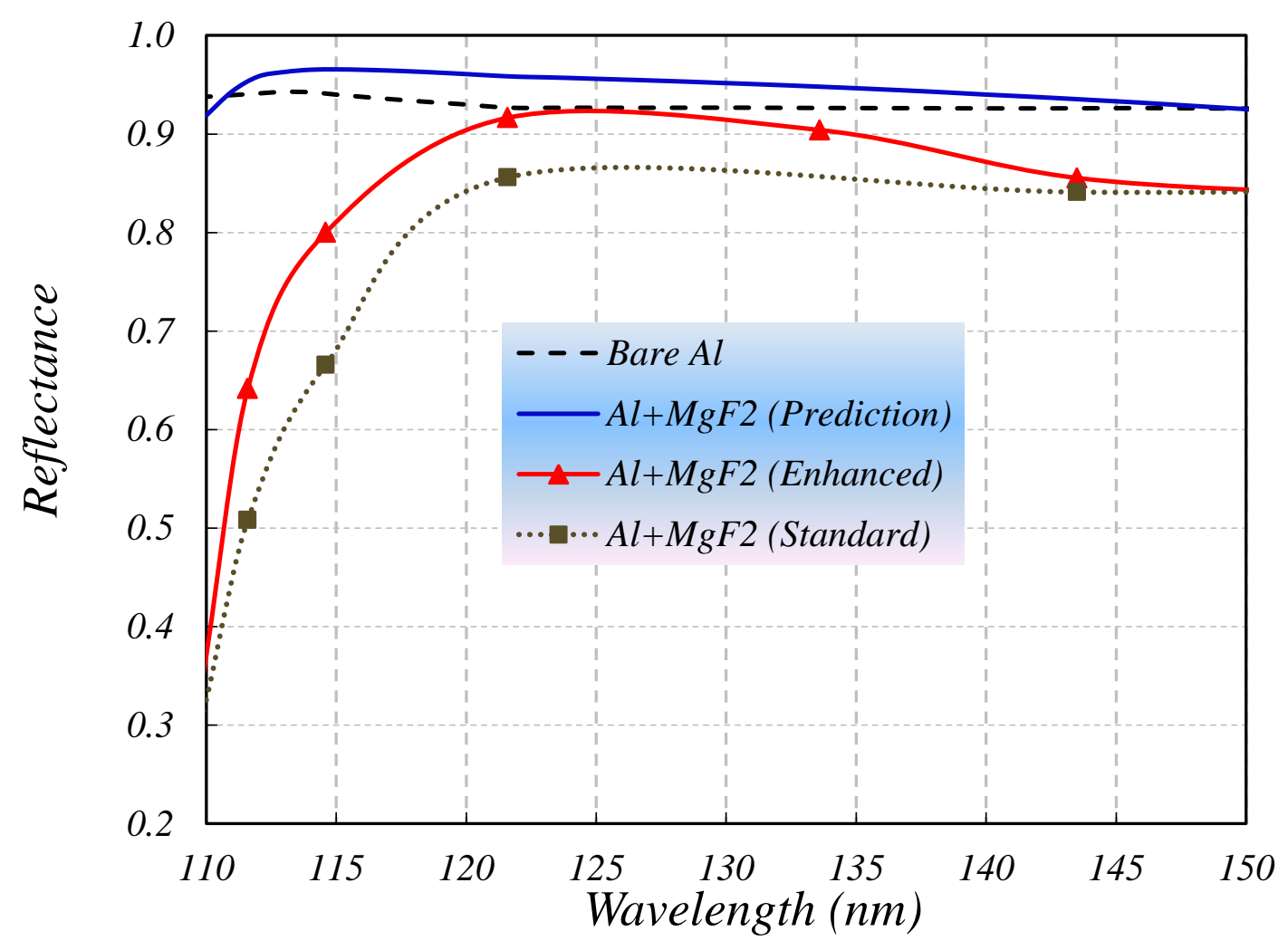

- Predicted vs. measured reflectance of bare $\mathrm{Al}$ and $\mathrm{Al}+\mathrm{MgF}_{2}$ reflectance (Al: $50.0 \mathrm{~nm}$; MgF2: 25.0nm)

- Enhanced performance is obtained by heating $\left(\sim 220^{\circ} \mathrm{C}\right)$ substrate during $\mathrm{MgF} 2$ deposition

- Reflectance is $>80 \%$ even at $115.0 \mathrm{~nm}$ 


\section{Evaporated Al+LiF Mirror Performance}

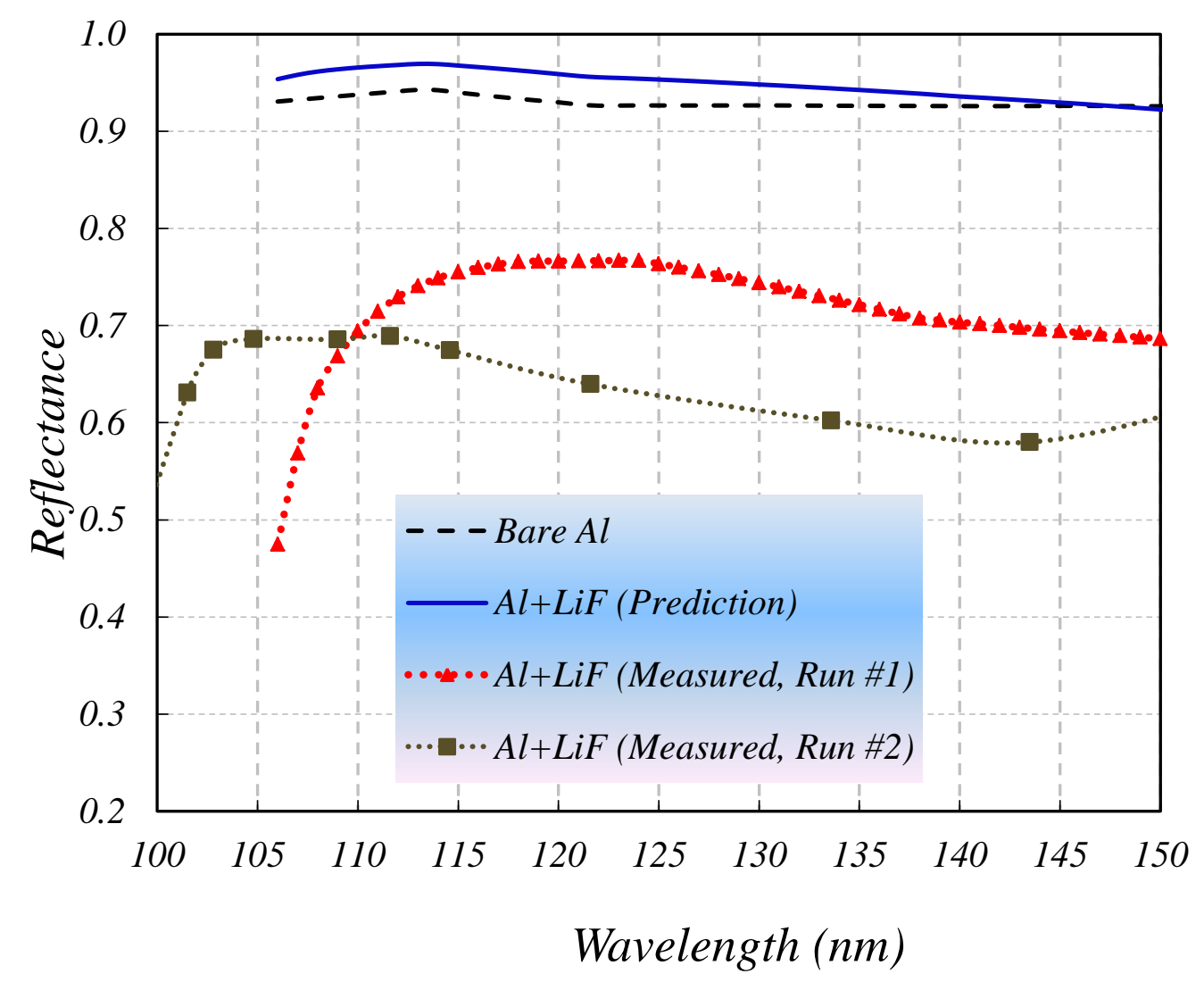

- Predicted vs. measured reflectance of bare $\mathrm{Al}$ and $\mathrm{Al}+\mathrm{LiF}$ reflectance

- Enhanced performance is obtained by heating substrate during LiF deposition. 


\section{Recent Results 1-meter Chamber}

Run AMC1207

Ref AMCT0902A

Wavelength (A)

*1216

1162

1216

1608

\section{Run AMC1208}

Wavelength (A)

*1216

1162

1216

1608

note:

* indicates initial measurement

0.823922691

Ref AMCT0902A

$* 0.811149486$

0.623358305

$\mathbf{0 . 8 1 4 5 5 9 7 4 9}$ slide AMC1207A center of 14" diam optic

* 0.863613442

0.655861189

0.863135491

0.881918126

0.815977285
AMC1208 3" from center of chamber

*0.890760365

0.809512748

0.896448174

0.843056068 slide AMC1207B 8" from center of optic

* 0.840455289

0.741373332

0.855357016

0.818922628 


\section{Throughput Performance for 2- and 4-meter Systems}

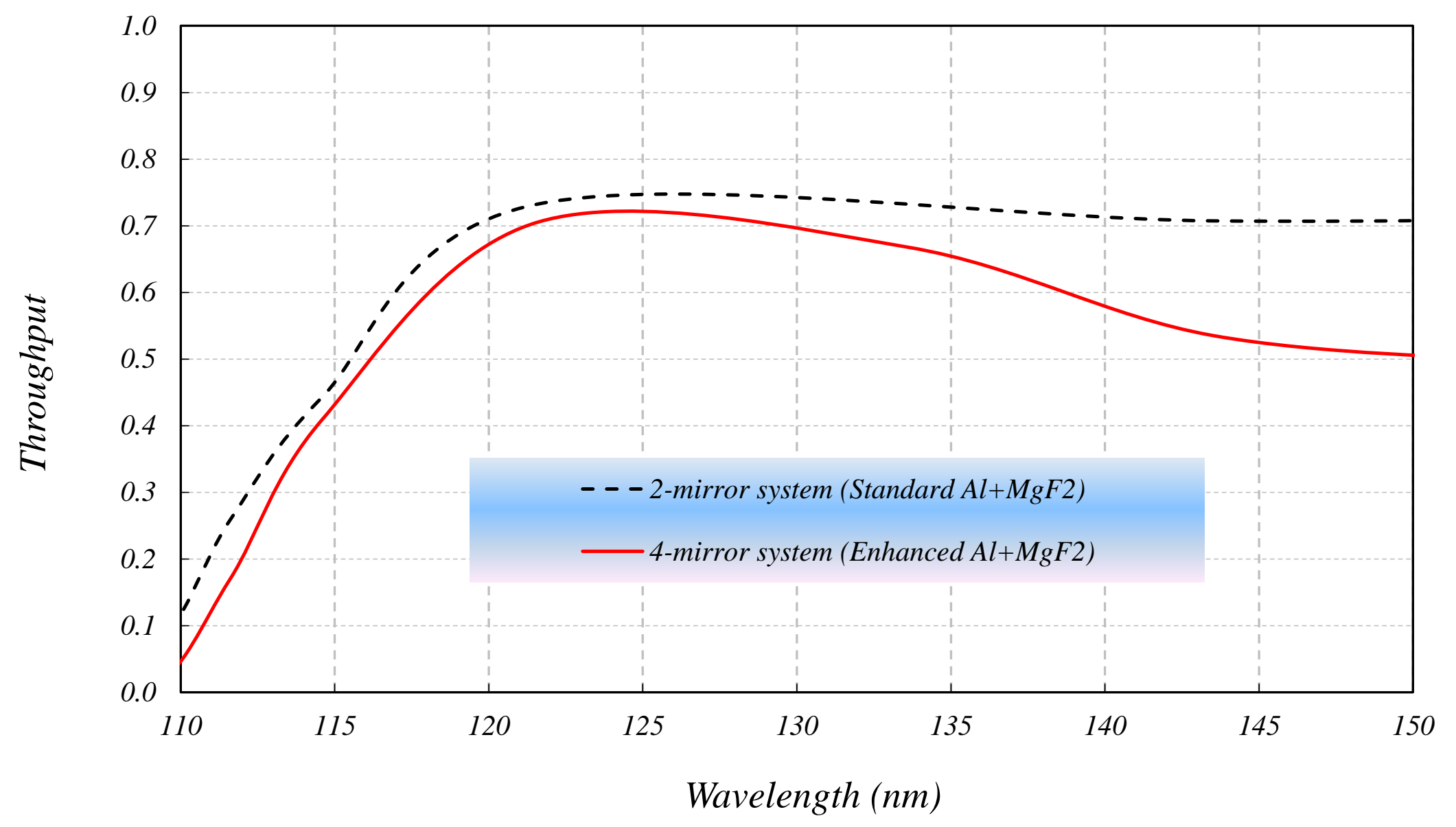




\section{GSFC 2-meter Coating Facility}

Design and fabrication of internal heat shields for 2-meter Chamber.

Optimized coating parameter for high FUV reflectance of a distribution of slides in center and out to a $\sim 0.5$ meter radius.

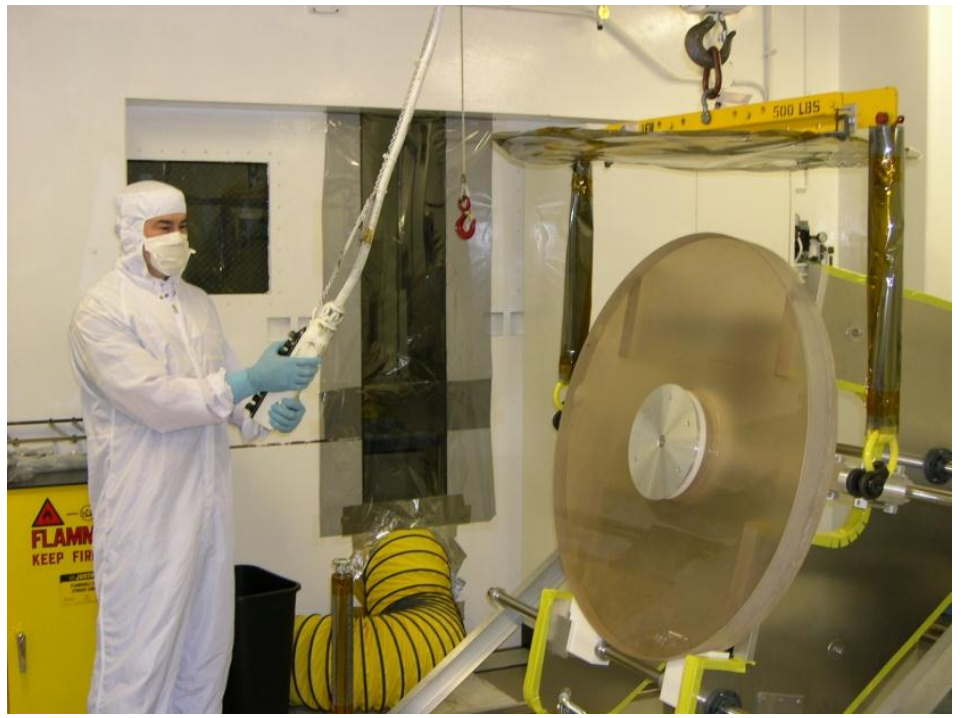

FIREBALL primary mirror loading

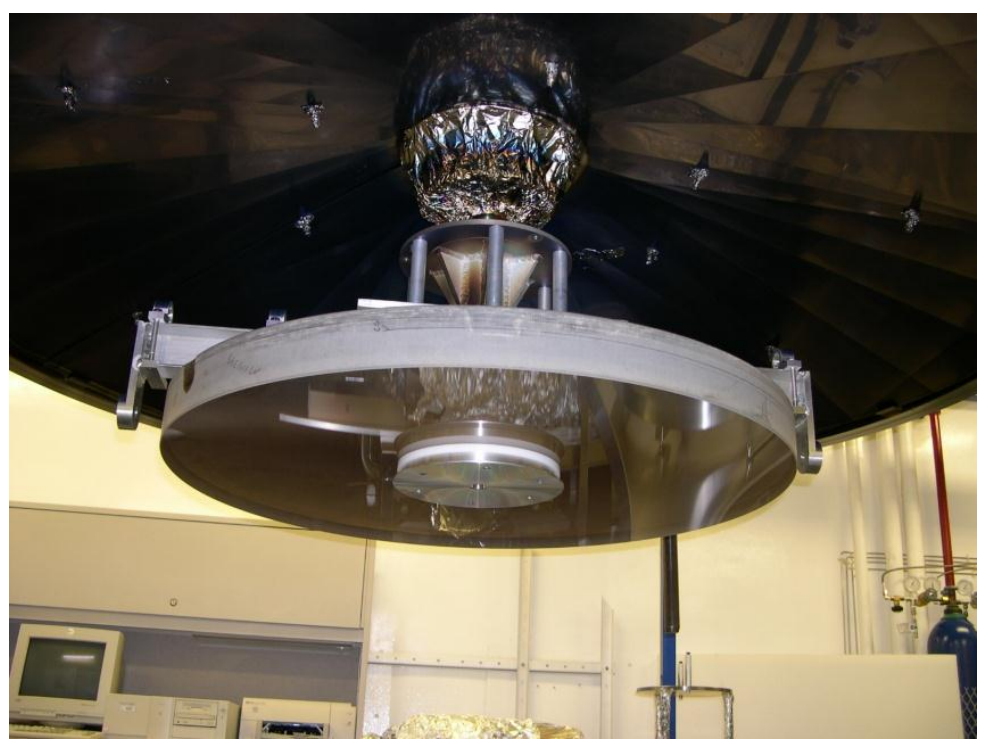

Mirror mounted in 2-meter chamber prior to coating 


\section{2-meter Chamber Heat Panel Concept}

- These wall panels were made out of stainless steel and were designed to easily interface with the existing internal configuration of the chamber.

- We are still in the design phase of the top shields for future installation in the chamber dome.

- We recently test fit the wall panels in the co.

\section{PANELS}

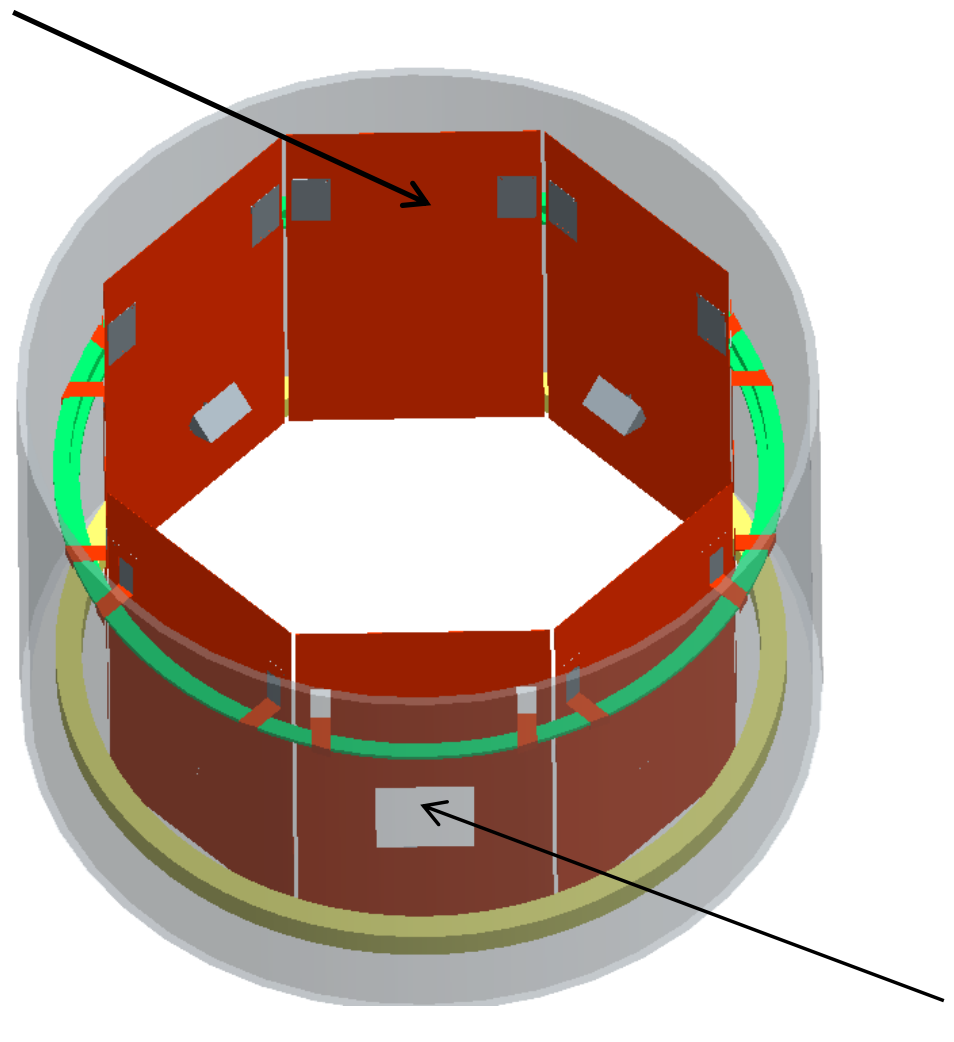

SINGLE

VIEWPORT

10" X 10"

SQUARE
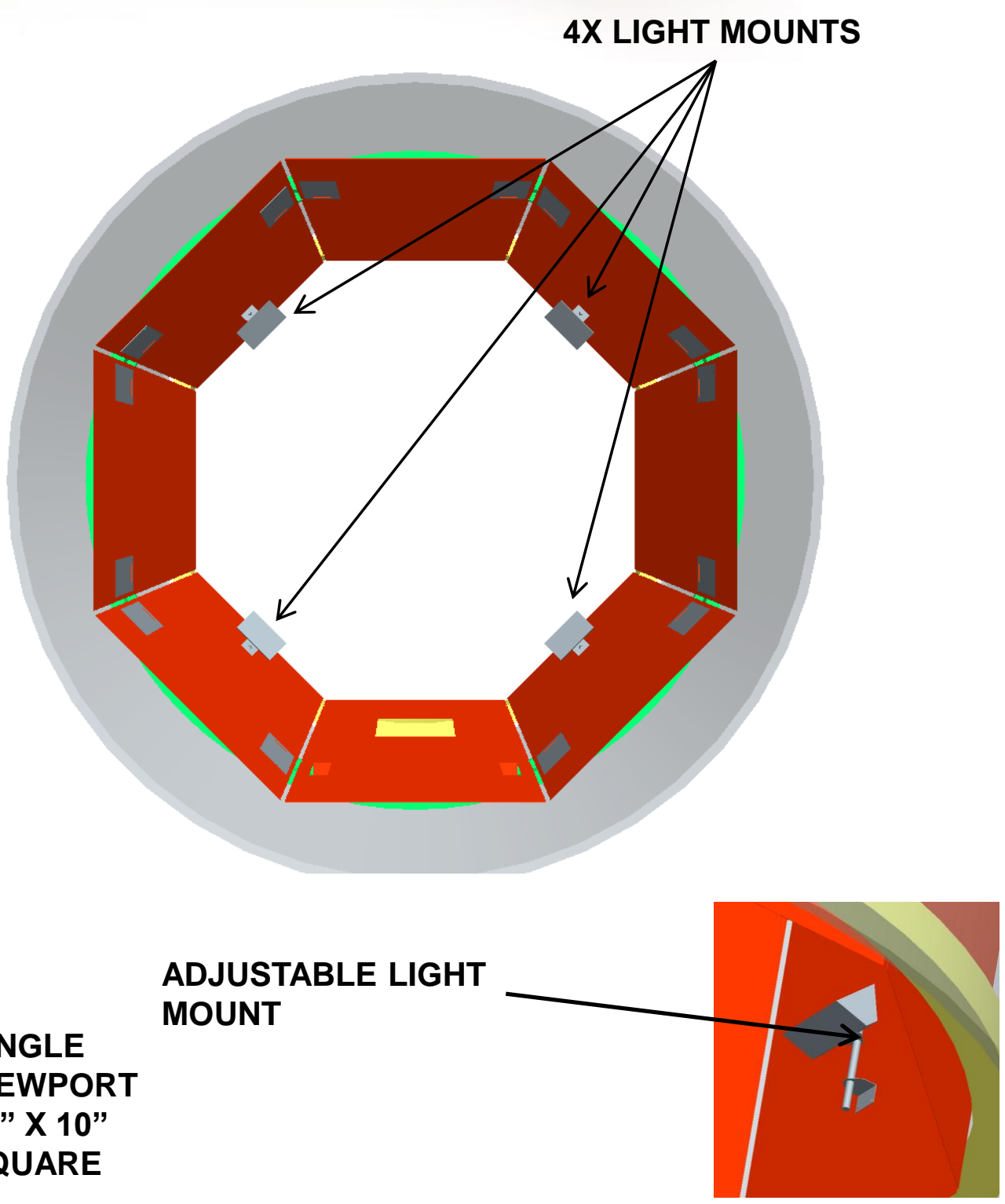

ADJUSTABLE LIGHT MOUNT

\section{S}




\section{Halogen-Quartz Lamp Heater Equipment}

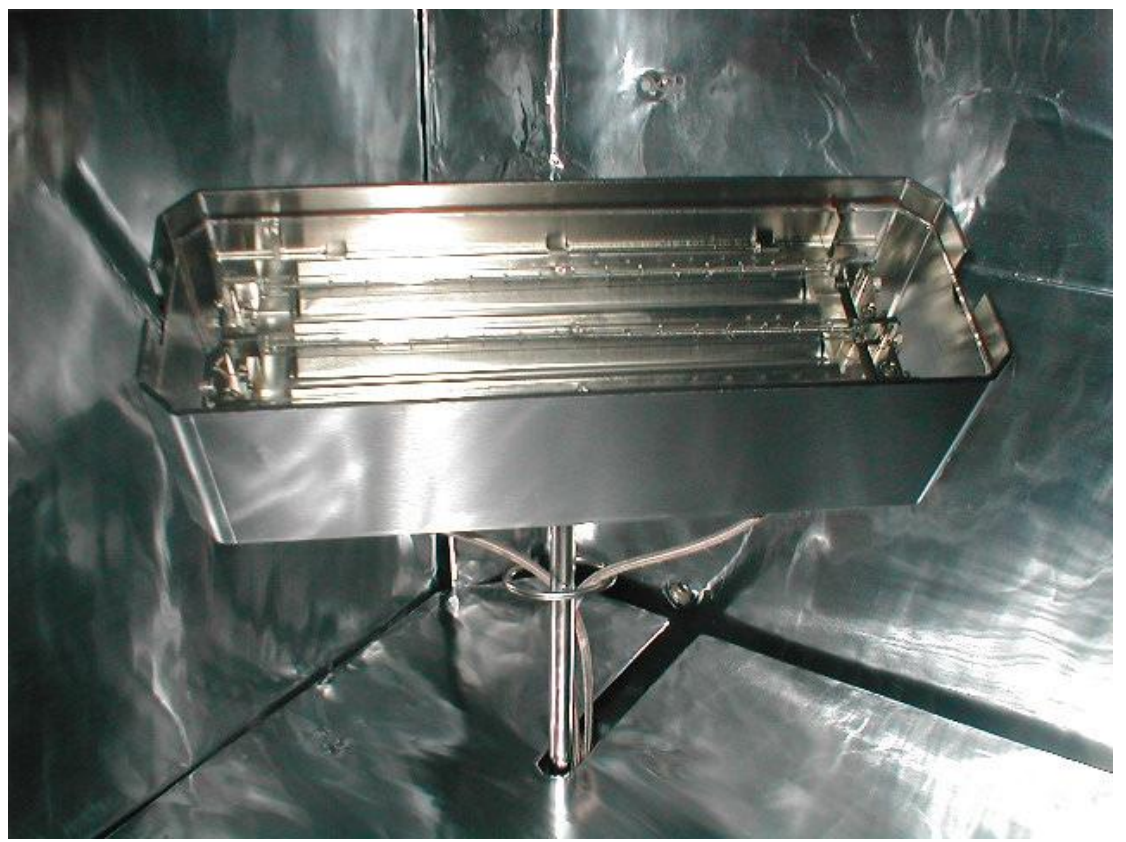

Quartz Lamp Heater Assembly

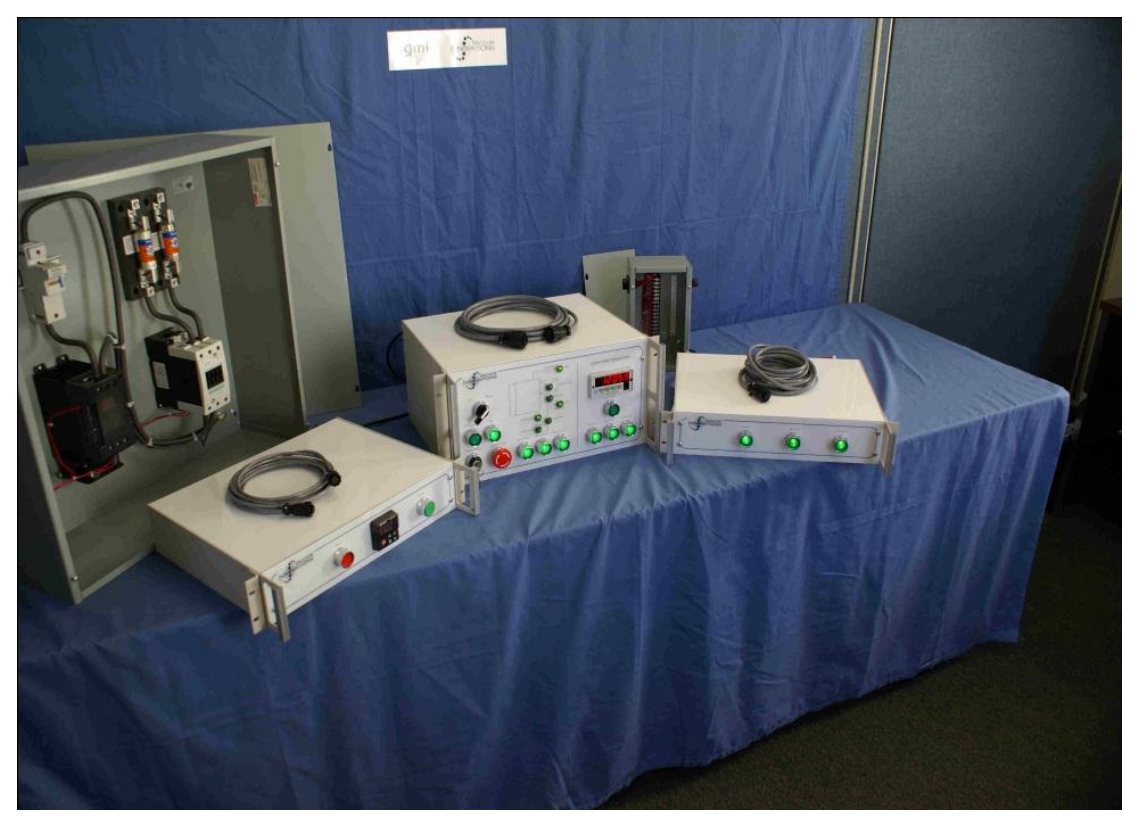

Power Supply System 


\section{FUV Reflecting Dielectric Coatings}

- Choose a high-index (H) and low-index (L)

$$
\text { pair combination }
$$

- Form a pair of $(H, L)$ layers with thicknesses equal to a Quarter-Wave Optical thickness at the design wavelength.

- $\quad$ Repeat the stack above until desired reflectance is achieved.

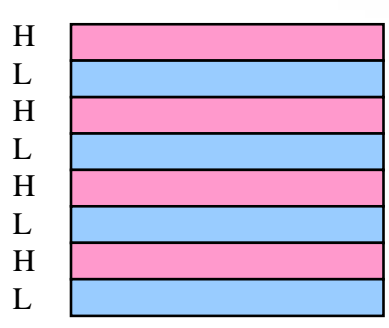

Note: The larger the difference between $\left(\mathrm{n}_{\mathrm{H}}-\mathrm{n}_{\mathrm{L}}\right)$ the better contrast and fewer layers needed to achieve a given $\mathrm{R}$

Options for coating materials:

$\mathrm{L}: \mathrm{MgF}_{2}(\mathrm{n} \sim 1.38)$

$\mathrm{H}:$ ?

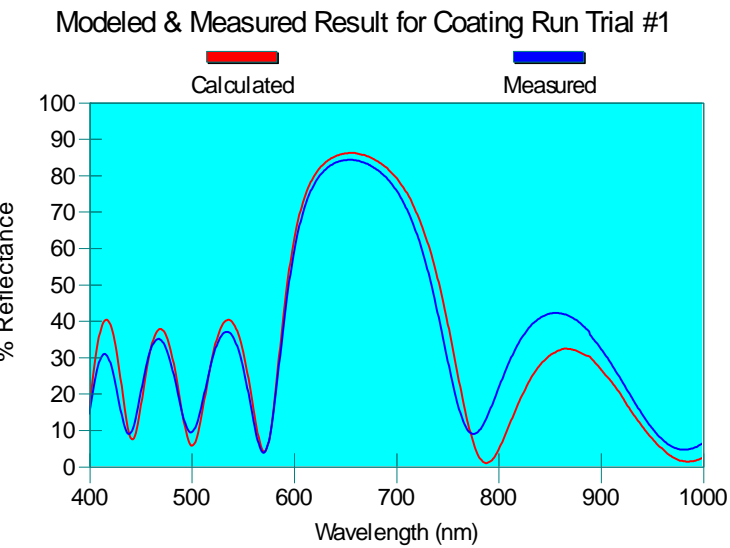




\section{Evaporation Details for Lanthanide Trifluorides}

\begin{tabular}{|c|c|c|c|c|c|c|}
\hline $\mathrm{LnF}_{3}$ & $\begin{array}{l}\text { VENDOR } \\
\text { \& FORM }\end{array}$ & PURITY & $\begin{array}{l}\text { PRICE } \\
\text { per } \\
\text { gram }\end{array}$ & $\begin{array}{l}\text { BOAT } \\
\text { (5 mil } \\
\text { canoe) }\end{array}$ & $\begin{array}{l}\text { RATE } \\
\AA \text { Åsec }\end{array}$ & $\begin{array}{c}\text { MELT } \\
\text { COMMENTS }\end{array}$ \\
\hline$\overline{\mathrm{PrF}_{3}}$ & $\begin{array}{c}\text { Cerac } \\
3-6 \mathrm{~mm} \text { fused } \\
1 / 8^{-1 / 4} \text { " pieces }\end{array}$ & $\begin{array}{c}99.9 \\
\text { vac. dep. } \\
\text { grade }\end{array}$ & $\$ 1.60$ & $\begin{array}{c}W \\
\text { (no Mo.Ta) }\end{array}$ & 6 & $\begin{array}{c}\text { bright green } \\
\text { smooth melt } \\
\text { large, shiny grains }\end{array}$ \\
\hline $\mathrm{SmF}_{3}$ & $"$ & $"$ & $\$ 1.62$ & Mo & 6 & $\begin{array}{l}\text { pale yellow } \\
\text { uneven melt } \\
\text { small, dull grains }\end{array}$ \\
\hline $\mathrm{EuF}_{3}$ & $"$ & $"$ & $\$ 16.00$ & $\begin{array}{l}\mathrm{Pt} \text { sheet } \\
2 \text { mil } \\
\text { (no Mo.Ta) }\end{array}$ & 4 & $\begin{array}{l}\text { light brown } \\
\text { smooth melt } \\
\text { large, shiny grains }\end{array}$ \\
\hline $\mathrm{GdF}_{3}$ & $"$ & $"$ & $\$ 1.70$ & Mo & 6 & $\begin{array}{c}\text { white, grey } \\
\text { uneven melt } \\
\text { small, shiny grains }\end{array}$ \\
\hline $\mathrm{TbF}_{3}$ & $"$ & $"$ & $\$ 7.50$ & $\mathrm{Ta}$ & 4 & $\begin{array}{c}\text { white, grey } \\
\text { smooth melt } \\
\text { small, shiny grains }\end{array}$ \\
\hline $\mathrm{DyF}_{3}$ & $"$ & $"$ & $\$ 1.60$ & $\mathrm{Ta}$ & 6 & $\begin{array}{l}\text { dark grey } \\
\text { smooth melt } \\
\text { small. shiny grains }\end{array}$ \\
\hline $\mathrm{HoF}_{3}$ & $"$ & $"$ & $\$ 2.30$ & $\mathrm{Ta}$ & 6 & $\begin{array}{c}\text { pink } \\
\text { smooth melt } \\
\text { large, shiny grains }\end{array}$ \\
\hline $\mathrm{ErF}_{3}$ & $"$ & $"$ & $\$ 1.90$ & $\mathrm{Ta}$ & 6 & $\begin{array}{c}\text { pink } \\
\text { smooth melt } \\
\text { large, dull grains }\end{array}$ \\
\hline $\mathrm{TmF}_{3}$ & $"$ & $"$ & $\$ 18.30$ & Mo & 6 & $\begin{array}{l}\text { white, grey } \\
\text { smocth melt } \\
\text { large, dull grains }\end{array}$ \\
\hline $\mathrm{YbF}_{3}$ & $"$ & $"$ & $\$ 2.40$ & $\begin{array}{c}\text { Mo } \\
\text { (no Ta) }\end{array}$ & 3 & $\begin{array}{l}\tan \\
\text { smooth melt } \\
\text { large, dull grains }\end{array}$ \\
\hline $\mathrm{LuF}_{3}$ & $\begin{array}{l}\text { Aesar } \\
\text { crystalline } \\
\text { lump }\end{array}$ & $\begin{array}{c}\text { " } \\
99.99 \\
\text { elec.grade } \\
\text { low oxy }\end{array}$ & $\begin{array}{l}\$ 37.00 \\
\$ 45.00\end{array}$ & $\begin{array}{l}\text { Mo } \\
\text { Mo }\end{array}$ & $\begin{array}{l}6 \\
6\end{array}$ & $\begin{array}{c}\text { white } \\
\text { whiter } \\
\text { smooth melt } \\
\text { large, dull grains }\end{array}$ \\
\hline
\end{tabular}

Ref.: Linda Jeanne Lingg, Ph.D. Thesis University of Arizona (1990). 


\section{Two FUV Candidate Materials: $\mathrm{GdF}_{3} \& \mathrm{LuF}_{3}$}
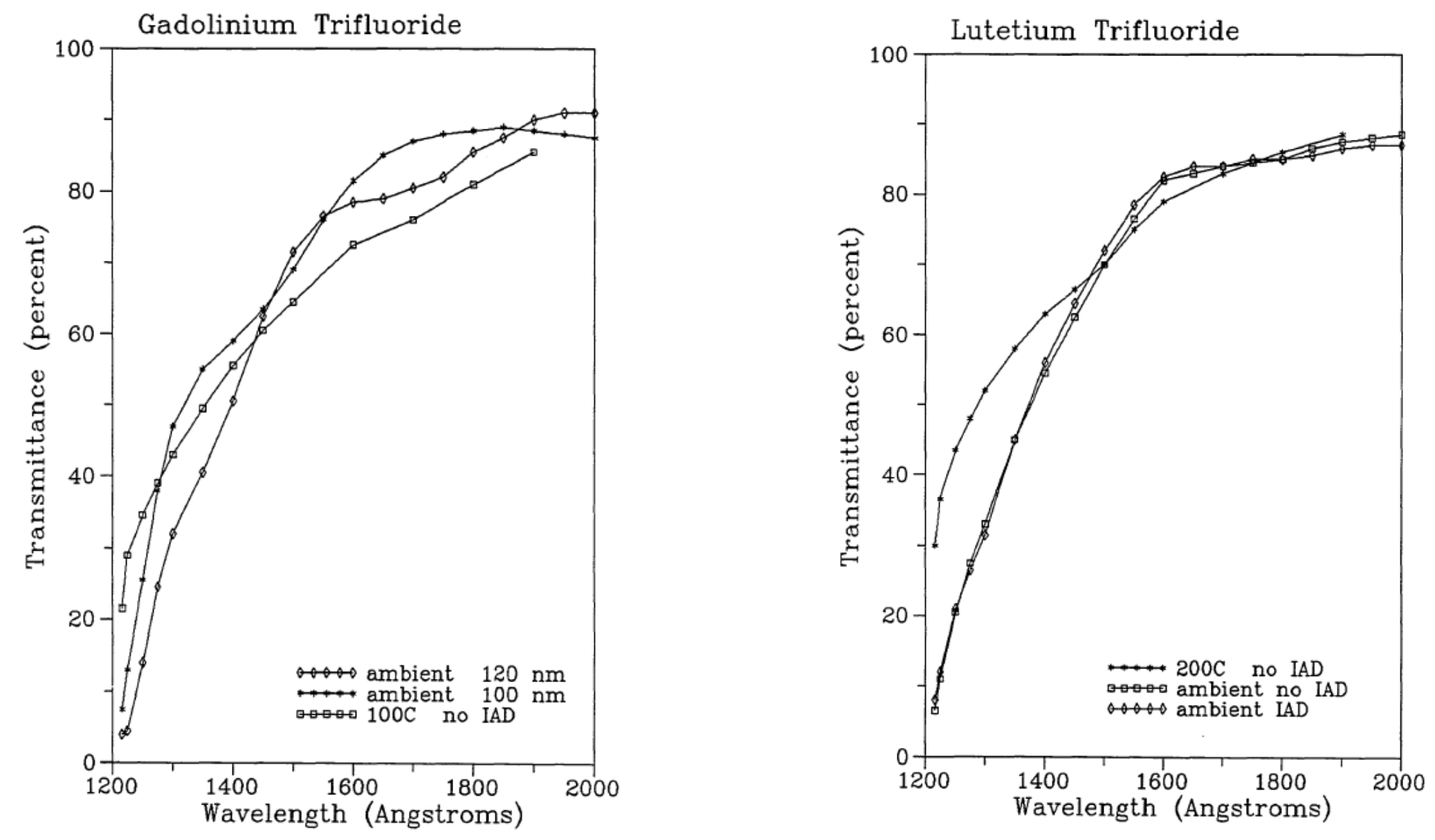


\section{Substrates Characterization}

Optical Transmission

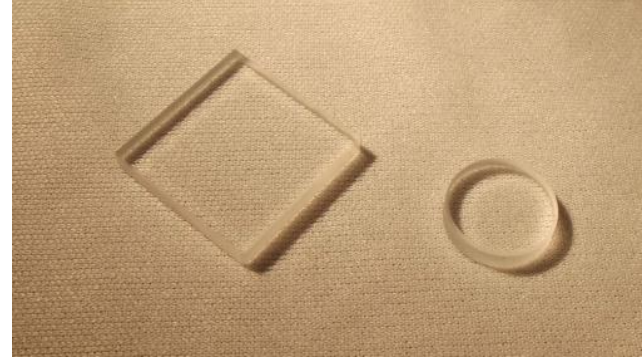

$\mathrm{MgF}_{2}$ \& LiF substrates have been procured

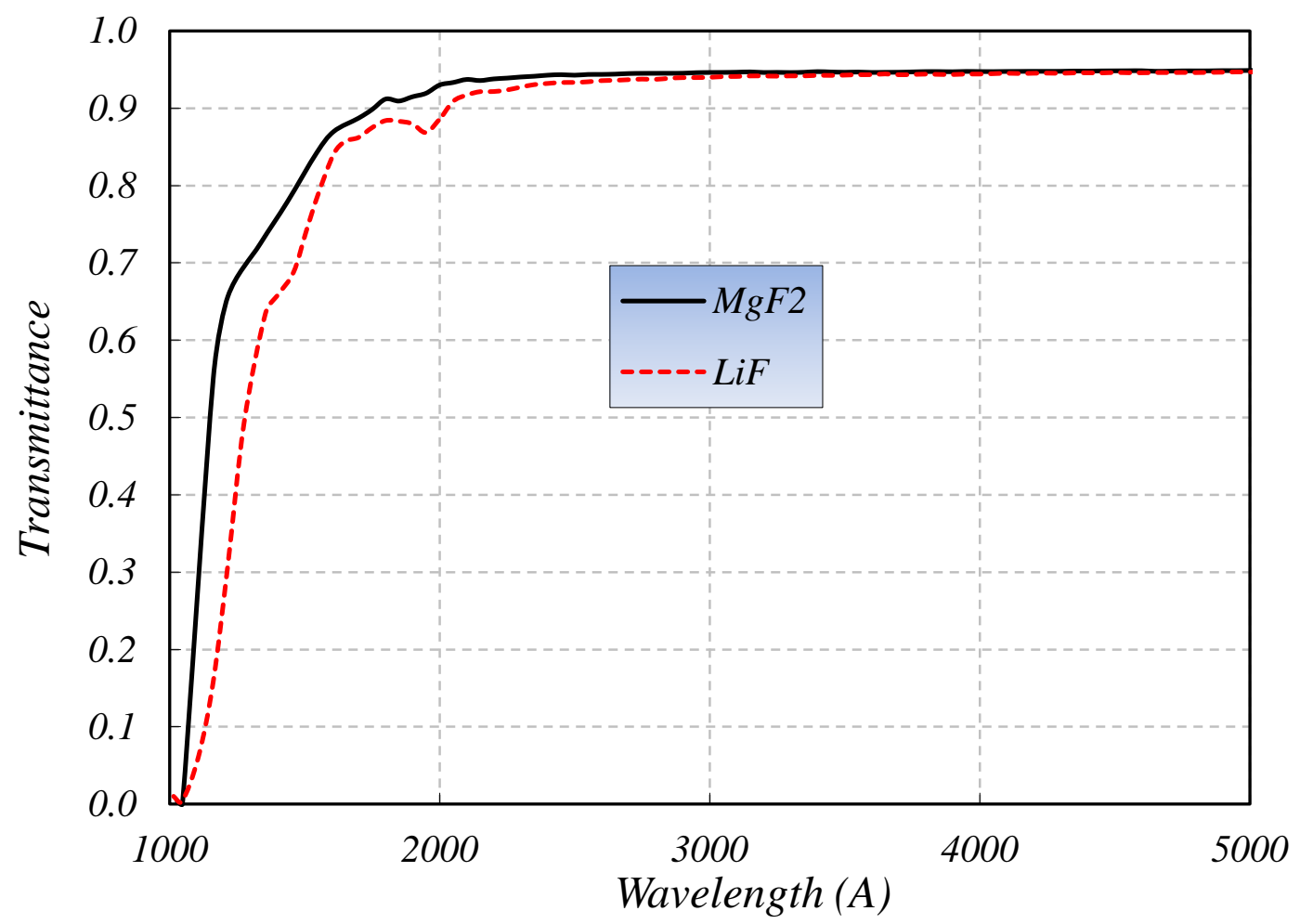




\section{GdF3 Film on MgF2 Substrate}

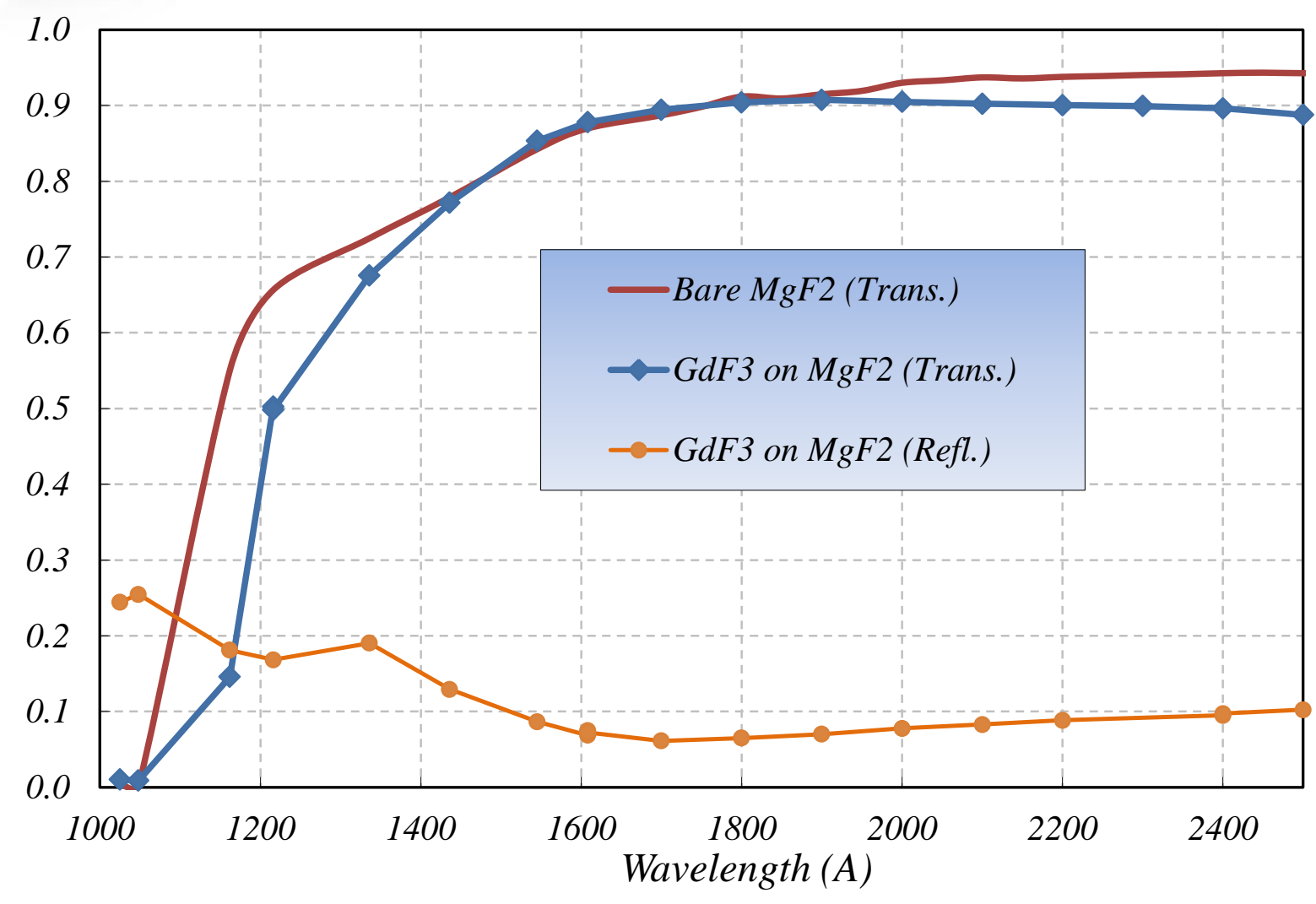

- 430 A GdF3 film on MgF2 substrate

- Measured T\& R will enable characterization of $n \& k$ 


\section{Ion Beam Sputtering Coating Chamber}

Upgrade chamber with a two-gas flow controller system.

$>$ Received shipment of Krypton gas cylinder to be used in the ion-beam sputtering deposition of $\mathrm{MgF} 2$ and LiF coatings.

$>$ Making preparation to start making test coating runs to "break-in" MgF2 targets.

$>$ Placed order of new magnets to refurbish ion gun in order to increase deposition rate.

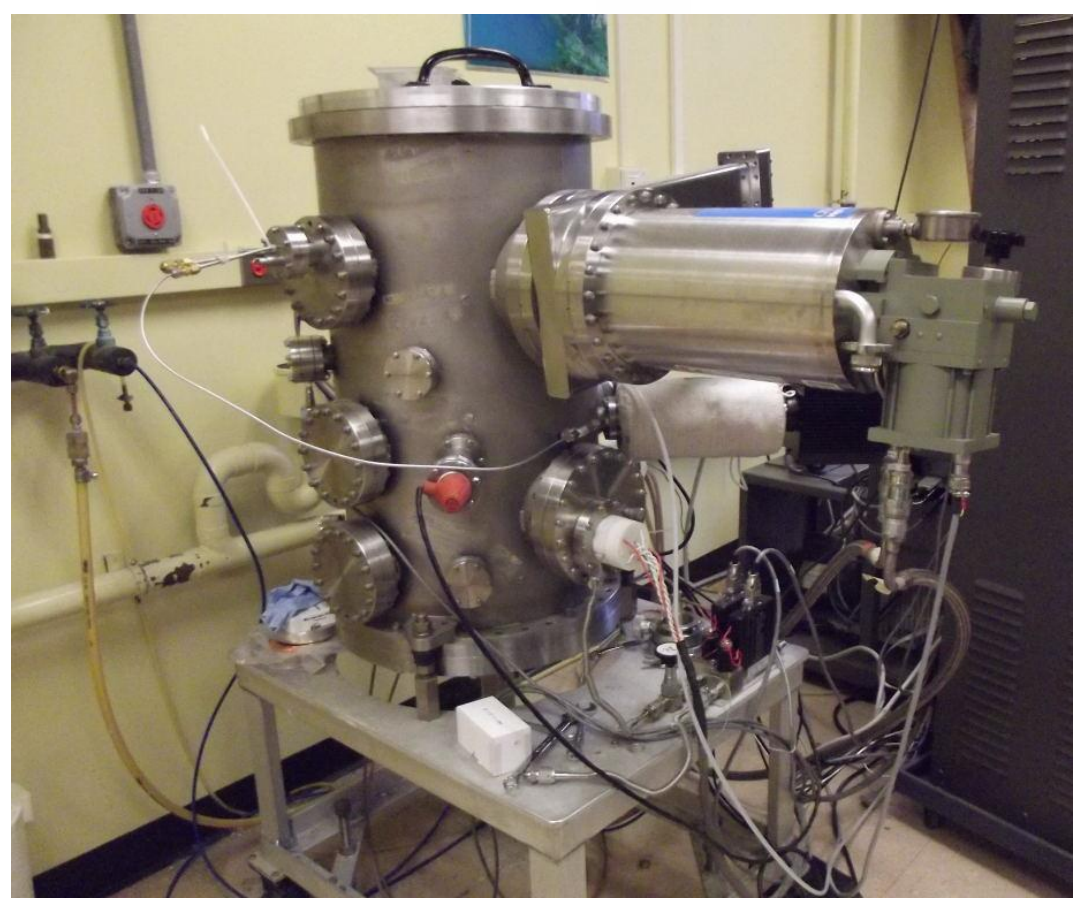




\section{Conclusion}

$>$ Reported gains in FUV reflectivity of $\mathrm{Al}+\mathrm{MgF}_{2}$ and $\mathrm{Al}+\mathrm{LiF}$ mirrors by employing a 3-step process during PVD coating deposition of these materials.

$>$ Preparations under way to transfer process to a large 2-meter chamber for allowing coating up to 1 meter diameter optics.

Characterization of lanthanide trifluoride material candidates to determine their FUV transparency for development of dielectric coatings.

Advantages of having better performing coatings will add more flexibility to a system design that is certain to improve overall performance.

$>$ Increasing system throughput is a very cost effective way to achieve more science and often is less costly than simply using a larger primary mirror. 


\section{Acknowledgements}

Steve Rice \& Felix Threat (Coating Technicians)

John Lehan (Materials Engineer)

Eric Mentzell (TTI Optical Designer)

John Sigwarth (TTI PI)

Funding agency: SAT grant from the NASA Cosmic Origin Program office 